\title{
Evaluating the Accuracy of Drug Dosing in the Prescriptions for Children under 5 Years Old from Non Pediatric Physicians in Outpatient Clinics
}

\author{
Ehab Mudher Mikhael* \\ Department of Clinical Pharmacy, College of Pharmacy, Baghdad University \\ *Corresponding author: ehab_pharma84@yahoo.com
}

Received August 11, 2014; Revised August 14, 2014; Accepted August 17, 2014

\begin{abstract}
Background: Pediatric patients are more vulnerable to the effects of a medication error and may experience a more serious adverse drug reaction than adults. Dosing errors represent the most common medication errors that occur in pediatric prescriptions. This study aimed to evaluate the accuracy of drug dosing that prescribed for children under 5 years by non paediatric specialist physicians in their outpatient clinic setting. Methods: An observational study was done in a single outpatient pharmacy located in Al - Amria at Baghdad/ Iraq from May to August 2014. 24 prescriptions to children less than 5 years old from non pediatric physicians were included in this study. Each prescription will be carefully examined to collect the following data: age and weight of the patient, diagnosis of the case, the name of the prescribed drugs with their strength, dose, route of administration and frequency of usage. All children were weighted in the pharmacy. Determination of the accuracy of drug dosing for each drug in all prescriptions was based mainly on drug dosing that stated in BNF for children. Results: The percent of correct dosing in the prescriptions of non pediatricians in their outpatient clinics to children below 5 years old is only $31 \%$. Dosing error was more common (90\%) in infants than in older children (64.5\%). antibiotics were the most common class of drugs that prescribed to children, while the highest percent of dosing error was related to steroid, antipyretic and analgesic prescribing. Conclusion: Dosing error is a common problem in the Rx of non pediatrician in outpatient clinics for preschool children in general and for infants specially.
\end{abstract}

Keywords: dosing error, pedatric

Cite This Article: Ehab Mudher Mikhael, "Evaluating the Accuracy of Drug Dosing in the Prescriptions for Children under 5 Years Old from Non Pediatric Physicians in Outpatient Clinics." American Journal of Pharmacological Sciences, vol. 2, no. 4 (2014): 61-64. doi: 10.12691/ajps-2-4-1.

\section{Introduction}

A medication error can be defined as 'a failure in the treatment process that leads to, or has the potential to lead to, harm to the patient [1]. Medication errors are common and include: prescribing errors, administration errors, dosing errors, inappropriate prescribing, inappropriate route of administration, prescription duplication or even equipment failure [2]. Medication errors can occur for any patient and at any age, and such errors have raised concerns about medication safety [3]. However pediatric patients are more vulnerable to the effects of a medication error and may experience a more serious adverse drug reaction than an adult, due to differences in weight or body surface area and because of the varying ability to metabolize and excrete medications [4]. Dosing errors represent the most common medication errors that occur in pediatric prescriptions $[5,6]$. That's why the dosages of most medicines that prescribed to children should be adjusted according to their ages and weights [7], to ensure administering not only the safe but also the effective dose of the drug. So this study aimed to evaluate the accuracy of drug dosing that prescribed for children under 5 years by non paediatric specialist physicians in their outpatient clinic setting.

\section{Methods}

A pilot observational study was done in a single outpatient pharmacy located in $\mathrm{Al}$ - Amria at Baghdad/ Iraq from May to August 2014. All prescription that were given to the responsible pharmacist by the pharmacy customers were examined to detect the age of the patient and the physician specialty. Any prescription to a child less than 5 years old from any physician except pediatricians was included in this study. A prescription will be excluded if the child was not present at the time of interviewing with the pharmacist.

Each prescription will be carefully examined to collect the following data: age and weight of the patient, diagnosis of the case, the name of the prescribed drugs with their strength, dose, route of administration and frequency of usage. All children were weighted in the pharmacy. If the diagnosis of the case was missed in the prescription, then the parents were asked about the 
diagnosis of their child case according to what did they were told by the examiner physician. Determination of the accuracy of drug dosing for each drug in all prescriptions was based mainly on drug dosing that stated in BNF for children [8].

Statistical analysis was done using Microsoft excel 2007. Discrete variables were presented as number and percents while continuous variables were presented as mean \pm standard deviation. Chi square test was used to test the statistical difference among discrete variables; chi square was measured by an internet based calculator. http://www.quantpsy.org/chisq/chisq.htm

$\mathrm{P}$ values less than 0.05 was considered significant.

\section{Results}

Table 1 showed the general information about the prescriptions ( $\mathrm{Rx}$ ) in this study at which 24 different $\mathrm{Rx}$ to children aged under 5 years old were examined, most $\mathrm{Rx}$ come from specialist physicians rather than GP physicians. Drugs in each $\mathrm{Rx}$ were ranged from 1 drug to a maximum of 4 drugs, with an average of 2.71 drugs. Total number of drugs that examined was 65 at which most of these drugs were given orally. Household measures were used in $98 \%$ of cases for the ordered medications. The weight of the child and the diagnosis of its case were absent in all Rx.

Table 2 showed that the average age of children in this study was around 2 years and their weight was slightly more than $12.5 \mathrm{~kg}$. The most common causes for seeking medical advice for children in this study were common cold and dermatological problems.

Table 3 showed that antibiotics were the most common class of drugs that prescribed to children, while the highest percent of dosing error was related to steroid, antipyretic and analgesic prescribing.

Table 4 showed that dosing error was more common (90\%) in infants than in older children (64.5\%). Drug over dosing occurred in $60 \%$ of cases in children below 1 year, and in $47 \%$ of prescribed drugs for children aged $1-5$ years. Under dosing for drugs also occurred more frequently in infants than in older children. Prescribing unlicensed or not recommended drugs occurred only in the $\mathrm{Rx}$ for children 1 - 5 years old. The percent of correct dosing in the prescriptions of non pediatricians in their outpatient clinics to children below 5 years old is only $31 \%$.

Table 1. General information regarding the examined prescriptions (Rx)

\begin{tabular}{|c|c|}
\hline Parameter & Value \\
\hline Total number of Examined Rx & 24 \\
\hline Rx from specialist physicians ( ENT, Dermatologist, ...) & 16 \\
\hline Rx from GP physicians & 8 \\
\hline Average number of drugs ordered in each Rx & $2.71 \pm 1.04$ \\
\hline Total number of ordered drugs in all Rx & 65 \\
\hline Number of ordered orally administered drugs N (\%) & $53(81.5 \%)$ \\
\hline Number of ordered parenterally administered drugs N (\%) & $5(7.7 \%)$ \\
\hline Number of not examined items (skin creams and nasal drops) N (\%) & $7(10.8 \%)$ \\
\hline Household measures used as a measure for orally administered drugs & $52(98 \%)$ \\
\hline Writing the diagnosis of the case in the Rx N (\%) & $0 / 24(0 \%)$ \\
\hline Writing the weight of the child in the Rx N (\%) & $0 / 24(0 \%)$ \\
\hline
\end{tabular}

Table 2. General information about the children and their diseases

\begin{tabular}{|c|c|}
\hline Parameter & Value \\
\hline Age of child in years & $2.06 \pm 1.1$ \\
\hline Weight of child in kg & $12.54 \pm 3.75$ \\
\hline Diagnosis of the child's case & $25 \%$ \\
Common cold and cough & $25 \%$ \\
Dermatological problems (impetigo, skin abscess and rash) & $17 \%$ \\
Diarrhea & $17 \%$ \\
Otitis media and other ear problems & $16 \%$ \\
\hline
\end{tabular}

Table 3. Classes of drugs that prescribed to children with their dosing accuracy

\begin{tabular}{|c|c|c|c|}
\hline Parameter & Total number of drug ordered & Doses administered correctly & Doses administered wrongly \\
\hline Antibiotic N (\%) & $30 / 58(51.7 \%)$ & $12(40 \%)$ & $18(60 \%)$ \\
\hline Antihistamines N (\%) & $12 / 58(20.7 \%)$ & $2(16.7 \%)$ & $10(83.3 \%)$ \\
\hline Cough and cold preparations N (\%) & $5 / 58(8.6 \%)$ & $2(40 \%)$ & $3(60 \%)$ \\
\hline Analgesic and antipyretics N (\%) & $3 / 58(5.2 \%)$ & $0(0 \%)$ & $3(100 \%)$ \\
\hline Antispasmodic and antiemetic N (\%) & $4 / 58(6.9 \%)$ & $2(50 \%)$ & $2(50 \%)$ \\
\hline Others (steroids and anti-flatulence) N (\%) & $4 / 58(6.9 \%)$ & $0(0 \%)$ & $4(100 \%)$ \\
\hline All drugs & $58 / 58(100 \%)$ & $18(31 \%)$ & $40(69 \%)$ \\
\hline
\end{tabular}

Table 4. Accuracy of drug dosing according to the child's age

\begin{tabular}{|c|c|c|c|c|c|}
\hline \multirow{2}{*}{ Parameter } & \multicolumn{3}{|c|}{ Wrong dose } & \multirow{2}{*}{ Correct dose } & \multirow{2}{*}{ P value } \\
\cline { 2 - 5 } & Over dose & Under dose & Drug is not recommended & & 0.038 \\
\hline Child under 1 year & $6(60 \%)$ & $3(30 \%)$ & $0(0 \%)$ & $1(10 \%)$ & 0.000 \\
\hline $1-5$ year & $23(47.9 \%)$ & $4(8.3 \%)$ & $4(8.3 \%)$ & $18(31 \%)$ & 0.000 \\
\hline All children & $29(50 \%)$ & $7(12 \%)$ & $4(7 \%)$ & $1 \%)$ & $1 \%$ \\
\hline
\end{tabular}




\section{Discussion}

There are many studies in the world specially in USA and European countries that focus on dosing error in pediatric patient in inpatient pediatric wards $[9,10]$, outpatient pediatric clinics [11] and pediatric emergency department [12], at which in all these cases the prescriptions were written by pediatrician, while there is no focus on dosing errors that done by non pediatricians except in very few studies, like a one which focus on errors that done by paramedics in pre hospital setting [13]. So the aim of this study was to explore the accuracy of drug dosing in the $\mathrm{Rx}$ of non pediatric physicians to children less than 5 years old in outpatient settings. On the other hand there is little research to detect and prevent medication errors which can result in significant morbidity and mortality to children in the Middle East countries and there is no any study in this regard in Iraq [14], and it may be that this study is the first one in this regard. This study focused on $\mathrm{Rx}$ for children under 5 years old, because Children $(<5$ years old) were at the highest risk of errors [15]. In this study most examined Rx were from specialist physicians rather than GP physicians which may be attributed to the higher availability of specialist physicians in outpatient clinics in Baghdad.

Drugs in each prescription were ranged from 1 drug to a maximum of 4 drugs, with an average of 2.71 drugs, this finding is different from that in Nigeria at which the numbers of drugs prescribed per patient per visit were between 1 and 8 with a mean value of 3.7 [16], this difference may be attributed to the difference in the age of children who participated in that study which was up to 12 years while in this study was up to 5 years.

The most common causes for seeking medical advice for children in this study were common cold, cough, dermatological problems followed by diarrhea. This finding is highly acceptable since common cold, cough and diarrhea among the most common diseases that affect pre - school children [17]; Furthermore skin infections like impetigo is a common dermatological disease that occur in children aged $2-5$ years [18].

The results of this study showed that antibiotics were the most common class of drugs that prescribed to children, similarly it was found that antibiotics are frequently prescribed to children especially in treatment of upper respiratory tract infections [19].

This study showed that dosing error is common and occur in $69 \%$ of ordered drugs in the $\mathrm{Rx}$ of non pediatricians in outpatient clinic setting, similarly the most common types of prescribing errors reported among the Middle Eastern countries were incorrect dose, wrong frequency and wrong strength, however the percentage of dosing errors that were reported during medication prescribing was ranged from $0.15 \%$ to $34.8 \%$ of the prescriptions [14]. These percentages are much lower than that found in this study which may be due to the different inclusion criteria of this study at which only prescriptions of non pediatric physicians were included while in those studies dosing error was measured for prescription to children from pediatric physicians. Additionally physicians in this study didn't measure patient weight, and it is well known that dispensing a pediatric prescription accurately requires additional information not commonly found on a prescription for an adult, most notably the weight of the patient [20]. Furthermore dosing of $98 \%$ of ordered drugs in the examined prescriptions were in household measures, and since household teaspoons measure anywhere from 2.5 to $7.8 \mathrm{~mL}$ (21) which means an additional dosing error may occur during the administration of the drug to the child by the parents.

Table 4 showed that dosing error occurred in $90 \%$ of ordered drugs for infants (below 1 year) while dosing error occurred in $64.5 \%$ of ordered drugs for children 1 5 years old. Similarly in Saudi Arabia dosing errors occur more frequently in infants than in older children (10). Drug over dosing occurred in $60 \%$ of cases in children below 1 year, and in $47 \%$ of prescribed drugs for children aged $1-5$ years. This may be because infants are usually requiring less dosing than older children on the basis of body weight and since body weight not measured by all participated physicians, additionally household measures were used in $98 \%$ of cases, so this may result in the higher percent of drug over dosing in infants rather than older children. Prescribing unlicensed or not recommended drugs occur in Rx for children 1 - 5 years old. Similarly in many other studies unlicensed drugs were usually prescribed to children (22).

Limitations of this study include a single pharmacy center for collection of the research data base with a small sample size, so further studies in many pharmacies at different sites of Baghdad with larger sample size are necessary to confirm the results of the current study.

In conclusion, dosing error is a common problem in the $\mathrm{Rx}$ of non pediatrician in outpatient clinics for preschool children in general and specially for infants.

\section{Reference}

[1] Jeffrey $\mathrm{K}$ Aronson. Medication errors: definitions and classification. Br J Clin Pharmacol. Jun 2009; 67 (6): 599-604.

[2] Tobaiqy M, Stewart D. Exploring health professionals' experiences of medication errors in Saudi Arabia. Int J Clin Pharm. 2013 Aug; 35(4): 542-5. Epub 2013 May 7.

[3] Patel I, Balkrishnan R. Medication error management around the globe: an overview. Indian J Pharm Sci 2010; 72: 539-45.

[4] Stuckey ER for the American Academy of Pediatrics Committee on Drugs and American Academy of Pediatrics Committee on Hospital Care. Prevention of medication errors in the pediatric inpatient setting. Pediatrics. 2003; 112 (2): 431-436.

[5] Miller MR, Robinson KA, Lubomski LH, et al. Medication errors in paediatric care: a systemic review of epidemiology and an evaluation of evidence supporting reducation strategy recommendations. Qual Saf Health Care. 2007; 16 (2): 116-126.

[6] Christiansen SR, Morgan JA, Hilmas E, et al. Impact of a prescription review program on the accuracy and safety of discharge prescriptions in a pediatric hospital setting. J Pediatr Pharmacol Ther. 2008;13 (4): 226-232.

[7] Taketomo CK, Hodding JH, Kraus DM: Pediatric \& Neonatal Dosage Handbook. 18th edition. Hudson, Ohio: Lexi-Comp; 2011.

[8] British Medical Association and the Royal Pharmaceutical Society of Great Britain. British National Formulary for children. 20112012 ed. UK: BMJ Publishing Group. 2011.

[9] Otero P, Leyton A, Mariani G, Ceriani Cernadas JM; Patient Safety Committee. Medication errors in pediatric inpatients: prevalence and results of a prevention program. Pediatrics. 2008 Sep; 122 (3): e737-43.

[10] Majed I Al-Jeraisy, Menyfah Q Alanazi and Mostafa A Abolfotouh. Medication prescribing errors in a pediatric inpatient tertiary care setting in Saudi Arabia. BMC Research Notes 2011, 4 294. 
[11] McPhillips $\mathrm{HA}^{1}$, Stille CJ, Smith D, etal. Potential medication dosing errors in outpatient pediatrics. J Pediatr. 2005 Dec; 147 (6): 761-7.

[12] Jost Kaufmann, Michael Laschat, Frank Wappler. Medication Errors in Pediatric Emergencies: A Systematic Analysis. Dtsch Arztebl Int 2012; 109 (38): 609-16.

[13] Hoyle JD, Davis AT, Putman KK, etal. Medication dosing errors in pediatric patients treated by emergency medical services. Prehosp Emerg Care. 2012 Jan-Mar; 16 (1): 59-66. Epub 2011 Oct 14.

[14] Zayed Alsulami \& Sharon Conroy \& Imti Choonara. Medication errors in the Middle East countries: A systematic review of the literature. Eur J Clin Pharmacol (2013) 69:995-1008.

[15] Folli HL, Poole RL, Benitz WE, Russo JC: Medication error prevention by clinical pharmacists in two children's hospitals. Pediatrics 1987, 97: 718-22.

[16] K A Oshikoya, H A Chukwura, O I Ojo. Evaluation of outpatient paediatric drug prescriptions in a teaching hospital in Nigeria for rational prescribing. Paediatric and Perinatal Drug Therapy, 2006; 7 (4).
[17] Hay AD, Heron J, Ness A; ALSPAC study team. The prevalence of symptoms and consultations in pre-school children in the Avon Longitudinal Study of Parents and Children (ALSPAC): a prospective cohort study. Fam Pract. 2005 Aug; 22 (4): 367-74. Epub 2005 May 16.

[18] Sarah Sahraoui, Oluwaranti Akiyode. Impetigo in Children and Adolescents. US Pharm. 2013; 38 (6): 68-71.

[19] Adam L. Hersh, Daniel J. Shapiro, Andrew T. Pavia, Samir S. Shah. Antibiotic Prescribing in Ambulatory Pediatrics in the United States. Pediatrics 2011; 128 (6): 1053-1061.

[20] Sandra Benavides, Donna Huynh, Jill Morgan, Leslie Briars. Approach to the Pediatric Prescription in a Community Pharmacy. J Pediatr Pharmacol Ther 2011; 16 (4): 298-307.

[21] Yaffe SJ, Bierman CW, Cann HM, et al. Inaccuracies in administering liquid medication. Pediatrics. 1975; 56 (2): 327-328.

[22] Lindell-Osuagwu L, Korhonen MJ, Saano S, Helin-Tanninen M, Naaranlahti T, Kokki H. Off-label and unlicensed drug prescribing in three paediatric wards in Finland and review of the international literature. J Clin Pharm Ther. 2009 Jun; 34 (3): 277-87. 